\title{
ALLOPURINOL IN GOUTY NEPHROPATHY AND RENAL DIALYSIS
}

\author{
BY \\ R. W. RUNDLES \\ From the Haematology Laboratory, Duke Hospital, and the Department of Medicine, \\ Duke University of School Medicine, Durham, North Carolina, U.S.A.
}

The pathogenesis of the renal disease associated with gout remains obscure in many details but the use of allopurinol for prophylaxis and possible treatment represents one of its most important applications. Nephropathy in gout may develop acutely but it is usually chronic, insidious, and slowly progressive. It is responsible for the death of 20 to 25 per cent. of all patients who have gout for periods of 5 to 15 years. Although a variety of vascular abnormalities and pyelonephritic changes have been described in the gouty kidney, the only unique pathological feature has been the presence of urate crystals. Interstitial tophi may obstruct collecting tubules and produce a reaction resembling pyelonephritis, or obstruction may actually predispose to infection. Recent anatomical studies, which have included biopsy as well as autopsy material, have led to the suggestion that nephropathy may be fundamentally a reaction of the kidney to an increased filtered load of uric acid. The effect of allopurinol in reducing the amount of uric acid excreted and preventing the development of nephrolithiasis eliminates major causes of renal injury (Rundles, Metz, and Silberman, 1966b).

An increased liability to form urinary urate stones parallels the development of hyperuricosuria, of course, in any disease state. In gout, the formation of urate stones is often a major manifestation of the disease and the one complication most amenable to correction by allopurinol therapy. Twelve of our patients with severe gout passed stones frequently, or had gravel and crystals in some instances almost daily; in all of them the formation and passage of urate stones was eliminated by allopurinol therapy.

Gouty nephropathy is a complication which we should aim to prevent by allopurinol therapy, for in most patients there is little evidence that advanced disease can be reversed once it has developed. In our present group of $\mathbf{5 5}$ patients with gout, some of whom have been on allopurinol therapy for as long as 4 years, none has developed new evidence of renal disease. In one exceptional patient, azotaemia and other manifestations of impaired renal function come and go in parallel with hyperuricaemia. Two patients with pre-existing gouty nephropathy have died of progressive renal failure despite prolonged allopurinol therapy. Three other patients had less impairment of renal function before allopurinol therapy was begun; two of them have had no evidence of further progression of renal disease, and in the third renal function seemed to improve slowly over a period of months (Rundles and others, 1966b).

The frequency of adverse reactions to allopurinol, for reasons we do not understand, appears to be greatest in patients with gouty nephropathy ando impaired renal function.

Hyperuricaemia is a frequent occurrence in patients with different types of chronic renal failure, and its aetiological importance or possible relationship to occult gout is often most difficult to assess.

In recent years, many patients with chronic renal failure have been treated with intermittent haemodialysis. Some of them have developed, in addition to anaemia and symptoms of peripheral neuropathy, what appears to be gouty arthritis in association with hyperuricaemia. During the past months two such patients have been available to us for study in reference to allopurinol therapy and drug metabolism (Hayes, Metz, Robinson, and Rundles, 1965). Both had virtually no renal function and were being maintained on a programme of chronic intermittent haemodialysis. Fairly good control of azotaemia and electrolyte balance was achieved by two 8-hour dialyses per week using a Kiil dialyser, but hyperuricaemia tended to recur quickly. One of the patients had an acute attack of arthritis resembling gout for which he was given 300 to $400 \mathrm{mg}$. allopurinol daily for several months. With the addition of allopurinol to his regimen, the serum uric acid level remained well below the range 
of normal between dialyses and he had no recurrence of joint symptoms. He had no evidence of drug reaction or other adverse effect from the allopurinol during the time he was treated.

An opportunity for making some observations regarding the metabolism of allopurinol (4-HPP) and of its oxidized derivative allo-xanthine (DHPP) was provided by one of these patients. The level of xanthine oxidase inhibition in his serum was assayed at various intervals before and after dialysis on several occasions. On a constant dose of 4-HPP, the concentration of inhibitor in the serum immediately before dialysis was equivalent to a level of 2.5 to $3.5 \mathrm{mg}$. DHPP per $100 \mathrm{ml}$. After each haemodialysis the level of inhibitor was reduced by 50 per cent. or more, and occasionally it appeared to be removed completely. The serum inhibitor level fell as blood passed through the dialyser. Analyses on fifteen occasions showed that DHPP was removed about as effectively as creatinine and uric acid with a clearance rate of approximately $80 \mathrm{ml} . / \mathrm{min}$. The level of DHPP in the serum was assayed during the course of maintained therapy on four occasions immediately after dialysis and then 12 hours to 4 days later before additional allopurinol therapy had been given. The level of serum inhibitor did not increase after dialysis in any instance and usually fell slightly (Hayes and others, 1965).

Some of the accumulated biochemical data which relates to the overall therapeutic efficacy of allopurinol is outlined in the Figure. The compound is well absorbed from the gastrointestinal tract. Little is lost in the urine since it is rapidly oxidized to alloxanthine by xanthine oxidase. The amount converted to other compounds, nucleotides or nucleosides, appears to be minimal. The oxidized product, allo-xanthine (DHPP), is also a stable and active inhibitor of xanthine oxidase. It is handled by the kidney much like uric acid and being maintained in the blood at a stable level undoubtedly affects the degree of therapeutic inhibition of xanthine oxidase (Elion, Kovensky, Hitchings, Metz, and Rundles, 1966a).
Earlier in this symposium, Dr. Savage suggested that we should put some emphasis on improving ouf classification, definition, and staging of gout fo $\overrightarrow{\mathrm{q}_{3}}$ didactic and therapeutic considerations. The follow ing may serve for preliminary discussion:

(1) Hyperuricaemia from underexcretion and $/ 8$ overproduction

(A) Asymptomatic

(B) With acute gouty arthritis

(C) With tophi or chronic gouty arthritis (ofte difficult to distinguish from rheumatoid arthritisdo

(2) HyPeruricosuria

(A) Asymptomatic

(B) With urinary urate stones, gravel, or crystalluri

(C) As above with obstructive nephropathy.

(3) Gouty NePhropathy

(A) Acute, non-obstructive

(B) Chronic, with or without infection.

The gouty diathesis varies, of course, in severit产 from patient to patient, and therapy should be designed with careful consideration for the expected duration of life. It is timely perhaps to discuss the re-evaluation of our total therapeutic approachô th gout in view of the newly available therapeutic agent:

Finally, we are approaching the time when plethora of agents and procedures which have been popular in treating the symptoms and objective abnormalities in gout can be prescribed on a more rational and physiological basis. An outline of current approaches to treatment may be set out follows:

\section{Therapeutic Agents in Gout}

(1) DIET: Less important in the modern era, but ex genous purine may be one factor precipitating acute attacks

(2) ColCHICINE: Reduction in phagocytosis or urate crystallization in tissues

(3) URICosuric DRUGS: Probenecid, sulphinpyrazore (Effect antagonized by salicylates)

ALLOPURINOL, 4-HPP
(4-Hydroxypyrazolo (3.4-d) pyrimidine)

XANTHINE OXIDASE

\begin{tabular}{|c|c|c|}
\hline Absorption from Gostrointestinal Tract 80 per cent. & 4-HPP riboside (troce) & Stable and Active Inhibitor. \\
\hline $\begin{array}{l}\text { Excretion in urine } \\
5 \text { to } 7 \text { per cent. in } 6 \mathrm{hrs}\end{array}$ & $\begin{array}{l}\text { Coniversion of 4-HPP } \\
\text { to DHPP } \\
\text { Ropid. I ro } 6 \mathrm{hrs}\end{array}$ & $\begin{array}{l}\text { Excretion in urine } \\
\text { Slow. } 60 \text { to } 70 \text { per cent. } \\
\text { in } 5 \text { to } 7 \text { days }\end{array}$ \\
\hline
\end{tabular}

Figure,-Biochemical data. 
(4) URICOSURIC AND ANTI-INFlammatory Agents: Phenylbutazone, oxyphenbutazone

(5) Anti-inflammatory Agents: ACTH, adrenal corticosteroids, indomethacin

(6) Xanthine Oxidase Inhibitor: Allopurinol. Decrease in uric acid production

We can offer no final opinion on what the role of allopurinol will eventually be in the standard therapy of gout, aiming to achieve the best long-term results with a minimum of risk, expense, and effort, but we can given an approximation which may serve for discussion purposes:

Tentatively it appears that for patients who have only an occasional episode of acute gout the symptomatic or prophylactic use of colchicine is probably sufficient. Uricosuric agents are effective in controlling hyperuricæmia in a great number of patients who tend to deposit urate in the tissues, but allopurinol may eventually prove to be equally or more effective. In patients with severe disease poorly controlled by standard means, and in those with impaired renal function, urate stones, and nephropathy, allopurinol is the agent of prime importance, best used in many instances in conjunction with colchicine. Determining the full extent to which allopurinol can be used to prevent renal involvement, joint destruction, and progressive increase in disease severity over the course of decades, will require careful long-term investigation (Rundles, Elion, and Hitchings, 1966a). 